COSMIC RAYS

Hobarl Conference

from a Correspondent

THE twelfth international conference on cosmic rays was held for the first time in the southern hemisphere at the University of Tasmania, Hobart, from August 16 to 25,1971 . In spite of the large distances involved for most participants delegates from twenty-two countries, not including Australia, attended.

In the high energy sessions the delegates heard of further experiments that had been carried out in the search for quarks and other particles. The contributions did not advance the position, however, and no conclusive new evidence for the existence of these particles was presented. The Sydney quark experiment has not been run significantly since the Budapest conference and no new events were reported. The experiment is currently being refurbished by the inclusion of five high pressure cloud chambers.

At the Calgary cosmic ray conference in 1967 , the University of Utah group reported that their underground measurements on the variation of cosmic-ray muon intensity with zenith angle, $\theta$, in the $\mathrm{TeV}$ range, showed no "sec $\theta$ " enhancement as predicted by theories involving pion parentage. It was suggested that this could be explained in terms of muon parents of very short lifetime, subsequently referred to as X-particles by the Utah group. The observations of the Utah group have continued and have been refined but the onset of the effect seems to have receded to somewhat higher energies and reaches a maximum, only to disappear again at even higher energies. Other experimenters, notably the group at the Tata Institute working in the Kolar goldfields, continue to find no evidence for a departure from the sec $\theta$ enhancement. It would seem that perhaps a third independent experiment at a depth of about $1,500 \mathrm{hg} \mathrm{cm}^{-2}$ should be carried out to try to resolve these apparently contradictory results.

Details of several large solid iron muon spectrographs being built or recently completed in order to study this effect directly were given at the conference. But the problems associated with the measurement of muon energies of about 3 to $5 \mathrm{TeV}$, coupled with the low flux of particles at these energies, mean that some time must elapse before these instruments will be able to contribute to an elucidation of this problem.

Although extensive air showers have been studied for several years, the origin and nature of the primary particles are not yet well understood. At the Jaipur cosmic ray conference in 1963 the results of the MIT (Volcano Ranch) group suggested that the slope of the integral primary energy spectrum changed from 1.6 (below $10^{16} \mathrm{eV}$ ) to 2.2 (between $10^{15}$ and $10^{18} \mathrm{eV}$ ) and back to 1.6 at energies greater than $10^{18} \mathrm{eV}$. These "ankles" were tentatively interpreted as reflecting a change in the mass composition of the primary cosmic-ray particles. The part of the spectrum beyond the second ankle was thought to be attributable to primary protons of extragalactic origin.

Perhaps the most significant change at the Tasmanian meeting was the report from the University of Leeds group working at Haverah Park in Yorkshire. These results showed no evidence for the existence of the second ankle. Furthermore, it was shown that by applying the same method of analysis to the MIT Volcano Ranch data, the evidence for the second ankle disappeared.

Preliminary data presented at the conference from the Sydney University Giant Air Shower Recorder (SUGAR) in the form of muon size spectra also showed no evidence for the second ankle at $10^{18} \mathrm{eV}$. Should these results be substantiated they will constitute an important observation which could lead to queries about the existence of extragalactic radiation. Measurements of the arrival directions of the most energetic showers yield no evidence of any anisotropy even when the galactic centre is overbead.

Several speakers described attempts to link the observed shower properties at sea level with the mass of the primary particles. Some progress in this field was reported by the Imperial College and University of Nottingham groups. It would seem that studies of the electron and muon component coupled with the observations of the radio emissions from showers might well be able to give more definitive answers.

Direct studies of the mass composition of the low energy part of the primary spectrum have continued. By using new techniques including superconducting magnets, knowledge of the light/ medium element ratio (L/M) has been extended up to $10,000 \mathrm{MeV}$ per nucleon. It has been found that the value is approximately 0.2 , and that the composition at the higher energies is equivalent to that at the lower energies.

Studies of elements with $Z>26$ have shown that the abundance of uranium is greater than expected. The disagreement between the values of $Z$ obtained from nuclear emulsion and plastic detectors has been resolved since the Budapest conference with the exception of one event. Studies of the light and heavy nuclei suggest that the age of the cosmic radiation is between $10^{6}$ and $10^{7} \mathrm{yr}$.

\title{
Arabinose Operon in vitro
}

IN Nature New Biology next week two groups, Zubay, Gielow and Englesberg and Greenblatt and Schlief, report the successful reconstruction in vitro of the regulatory system which controls the expression of the arabinose operon of Escherichia coli. Their experiments are notable, for this is the first time that a positive regulator protein which causes the expression of a specific operon has been made to function in vitro. Furthermore, these in vitro experiments confirm the picture of the regulation of the arabinose operon which has emerged from studies of intact $E$. coli and they provide a method for assaying for the elusive arabinose regulator protein.

Zubay and his colleagues used the cell free system developed by his group to support the regulated expression of the lac operon to define the elements required for the synthesis in vitro of L-ribulokinase when the system is programmed with phage $\theta 80$ dara DNA which includes the arabinose operon. They find that in addition to cyclic AMP, the activator protein which binds cyclic AMP and ppGpp, cell extracts containing the protein specified by the ara $\mathrm{C}$ gene are required together with arabinose. Genetic analyses had indicated that the araC gene protein can act both as a repressor and an activator of the arabinose operon and these in vitro experiments confirm the latter function of this protein.

Greenblatt and Schlief have, like Zubay and his colleagues, exploited mutants of the araC gene to prove that the araC protein is an activator in vitro of the arabinose operon. Moreover, they have shown that under certain conditions the ara $\mathrm{C}$ protein can act in vitro as a repressor of the arabinose operon. These findings fully confirm the idea that the araC protein can exist in two functional states, repressor and activator, in equilibrium and that an inducer such as arabinose favours the activator form. Greenblatt and Schlief have also reached the interesting conclusion that the protein specified by constitutive mutants of the araC gene can activate the arabinose operon in vivo in the absence of arabinose not because it is "frozen" in the activator state but because it can respond not only to arabinose but also to some as yet unidentified metabolite always present in growing cells.

Attempts to purify the araC protein by virtue of its ability to bind to arabinose or arabinose operon DNA have all failed, but now that the cell free system for ribulokinase synthesis provides an assay for this protein, its purification can be anticipated and that will open the way to a detailed analysis of a regulatory molecule. 